\title{
Integrated urban modeling: Past, present, and future
}

\author{
Eric J. Miller \\ University of Toronto \\ miller@ecf.utoronto.ca
}

\section{Article history:}

Received: August 3, 2017

Accepted: October 11, 2017

Available online: June 11, 2018

\section{Introduction}

At the 2014 World Symposium on Transport and Land-Use Research a well-attended workshop was held that presented a snapshot of the current state of operational practice of integrated urban models (IUMs), which includes numerous operational applications of a variety of model systems worldwide. A 2013 review of the integrated modeling state of practice found that approximately 200 model systems involving at least 40 reasonably distinct frameworks have been developed over the past 40 years, with somewhere in the order of 20-40 implementations in active use at the time of the review (HBA Specto, 2013). Most of this modeling activity has occurred in the United States, Western Europe (including the UK) and South America. Some convergence in methods is evident in terms of an increasing number of applications of a relative handful of commercial software packages (notably UrbanSim, PECAS, Tranus, DELTA and Cube Land ), although these model systems typically differ methodologically in

Copyright 2018 Eric J. Miller

http://dx.doi.org/10.5198/jtlu.2018.1273

ISSN: 1938-7849 | Licensed under the Creative Commons Attribution - Noncommercial License 4.0

The Journal of Transport and Land Use is the official journal of the World Society for Transport and Land Use (WSTLUR) and is published and sponsored by the University of Minnesota Center for Transportation Studies. This paper is also published with additional sponsorship from WSTLUR. 
significant ways.

IUMs have been long criticized for their "data-hungriness," "complicatedness" and numerous other technical challenges (Lee, 1973), as well as for their theoretical short-comings (Lee, 1973, 1994; Timmermans, 2003). Certainly their checkered history testifies to:

- The very significant technical challenges involved in developing such complex, large model systems

- Several historical failures to "deliver the goods" in terms of robust policy analysis and decision support capabilities

- A historical disinterest within urban planning and decision-making in transport/land-use interactions

- And, hence, an unwillingness to invest in these methods

At the same time, continuing, very significant technological advances in computer hardware and software capabilities, GIS and large-scale urban spatial databases, and model estimation software have made possible the development, implementation and use of such models in operational planning settings in various countries worldwide. As noted by the review cited above, it is indeed a practical proposition to build and use such models, Lee's criticisms notwithstanding.

Further, as Miller (2009) has argued, the theoretical foundations for IUMs are, in fact, reasonably strong. These foundations include:

- Very strong microeconomic models of housing markets (bid choice: Martinez, 1992; Rosen feld, Chingcuanco, \& Miller, 2013)

- Strong models of regional spatial economic processes and interactions (the "MEPLAN family" of models: MEPLAN, Tranus, PECAS)

- Very well developed, robust random utility/discrete choice modeling theory and methods t hat can be applied to many/most spatial processes

- The agent-based paradigm for socioeconomic modeling which provides a very flexible, powerful framework for modeling complex, heterogeneous behaviors

Most importantly, there is a steadily growing recognition within the urban policy field that the transport/land-use interaction does exist and does matter. The 21st Century is the century of the city, with more than $50 \%$ of the world's population now living in urbanized areas. Existing metropolitan regions continue to grow in size, while new cities are continuously being developed. There are now 37 urban areas of 10 million or more people (Demographia, 2017), with this number certain to grow in the coming decades. The challenges which this unprecedented urban growth is posing for urban design and urban transportation systems are profound. It is well understood that transportation technologies and systems have shaped urban development and functioning through history. If the world is to have any hope of achieving some form of economic, social and environmental sustainability then we must do a better job of integrating land use and transportation system design in holistic and comprehensive ways (Miller, 2013a).

Specifically, "transportation problems" of excessive congestion, equitable accessibility to jobs, services, etc., public health, environmental impact (both pollution and, even more critically, greenhouse gas emissions), and economic productivity and competitiveness are not solely addressable through transportation policies and investment alone. The physical design of the city-at both the "macro" scale of regional metropolitan design and the "micro" scale of neighborhood design—critically interfaces with transportation technological capabilities to determine the physical and economic feasibility of alternative transportation network designs, particularly transit and active (walk/bicycle) travel systems and the demand for and performance of the transportation system.

Further, the benefits and costs of expensive transportation infrastructure investments cannot be 
appropriately assessed if the "feedback" effects of these investments on urban form, land values, property taxes, quality of life, other infrastructure needs, etc. are not quantified and included in evaluation and decision-making. Certainly, in the North American case it is highly arguable, for example, that the benefits of investment in new public transit services are significantly underestimated due to failure to consider the potential land value and urban development impacts of these investments. At the same time, the adverse impacts of highway investments in terms of increased "urban sprawl"- excessive consumption of valuable land, increased need for investment in other expensive physical (water, sewers, solid waste, etc.) and social (hospitals, schools, etc.) infrastructure, etc.- -are also underestimated, if they are considered at all. The result of this very incomplete assessment of the land-use implications of transportation investments is (again at least within the North American context) an almost universally systematic under-investment in transit and over-investment in highways. The "full costs" of post-WWII suburban development are in many municipalities only now becoming apparent, not only in terms of the dysfunctional performance of the overly-congested road networks upon which they are predicated, but in terms of excessive maintenance and rehabilitation costs of aging infrastructure overlaid upon tax bases that are often stagnant or shrinking.

IUMs clearly offer the potential to significantly reduce this decision-making myopia by expanding the scope of the analysis, evaluation and debate concerning transportation system design and investment to include major impacts and feedbacks between the transportation system and both urban spatial form and the urban regional economy. Specifically, currently operational integrated urban model systems are capable of modeling, to varying degrees:

- Demand, supply and endogenous price formation in urban housing and commercial floor space markets

- Household demographics

- Urban spatial economic exchanges (urban labor markets and, sometimes, the internal production of goods and services)

- Household auto ownership

Given this abstract case for IUMS, the question arises concerning why they are not used more often in operational practice, and what, if anything, can research do to address this issue? Section 2 of this paper explores the first of these two questions, while Sections 3 and 4 lay the foundation for a proposed new research "push" to improve the operational viability and use of IUMs.

\section{$2 \quad$ Are we"in the doldrums"?}

Three dozen or so current integrated model system implementations worldwide is hardly a major success story. It also does not represent a major growth trend over the past two-plus decades. Wegener (1995), for example, identified 20 major "urban modeling centers" worldwide and reviewed 13 different models, not all of which were in operational planning practice. Miller, Kriger, and Hunt (1998) similarly identified 6 major operational model systems for detailed review, most of which still define the today's operational state of practice.Iberatia sunt, ommolora voloruptatem que nulliqui dem net molor sit, cuptas ut plam, sequae sitio comnimeni di deribus acero duntoreria debitis eiumenecus.

Indeed, arguably the only major model systems that have emerged since the mid-1990s are PECAS and ILUTE (and ILUTE is not yet in operational practice). And both of these derive from design concepts identified in Miller, et al. (1998), as well as the Oregon TLUMIP program of the late 1990s and early 2000s (Weidner, Abraham, Donnelly, Hunt, \& Freedman, 2007). Also, as alluded to above, PECAS' conceptual foundation explicitly lies within the MEPLAN/TRANUS, spatial input-output framework which dates to de la Barra’s PhD thesis (de la Barra, 1989). A very important exception to this generalization is the work of Wegener and his colleagues in Germany, who continue to advance the 
field of microsimulation-based land-use models (Strauch, et al., 2005; Spiekermann \& Wegener, 2007; Moeckel, Schwarze, Spiekermann, \& Wegener, 2007; Wegener, 2011; Moeckel, 2017). The Cube Land software is also of more recent vintage, but it is simply a commercial implementation of Martinez MUSSA model, which dates to the early- to mid-1990s in vintage.

In a seminal paper, Pas (1990) asked the question of whether activity-based travel models were at that time "in the doldrums," given the lack of operational implementation of a seemingly behaviorally strong and policy sensitive modeling approach. Pas ended his review on an optimistic note, concluding that the activity-based approach was still the best way forward for developing improved travel models, a judgement which now seems vindicated (although it took another 10-15 years for these models to achieve widespread adoption, a process that is still on-going today).

It seems appropriate to ask this same question of integrated urban models. Considerable evidence exists to suggest that they are, indeed, in the doldrums (at least with respect to operational implementation). Perhaps most importantly, as noted above, these models have failed to achieve widespread adoption. Over time some urban regions have implemented a new model system, but others over time have abandoned their systems. As a result, there has been little net growth in usage, despite some local "success stories."

Also, as already noted, currently operational model systems are largely based on $20+$ year-old designs. Incremental improvements in these models, of course, have occurred. These largely consist of increased spatial and socioeconomic disaggregation, exploitation of state-of-the-art GIS technology and datasets, improved computational efficiency, etc. These are all useful improvements, but they hardly represent a breakthrough in model formulation.

As discussed in detail by Timmermans (2003), operational models are overly static and overly dependent on rather simple logit choice models. There is nothing wrong with using a logit model in an appropriate application. The complaint is that operational implementations do not capture market dynamics, they are not sufficiently context-sensitive, and the basic model formulation hasn't changed in 25 or more years. Although not usually acknowledged as such, they are generally static equilibrium formulations that, at best, capture the cross-sectional distribution of (for example) a housing market at the time of model calibration. They are not models of dynamics/process, nor is it clear that they adequately capture market interactions and price formation.

Further, models of building/floor space supply have shown very little development over the decades. Arguably, weak models of building supply represent the single biggest limitation of current models. The lack of improvement in this component of model systems is particularly distressing given its importance with respect to policy analysis and forecasting: if we can't get the buildings in the "right place" then we have little chance of currently predicting future year population and employment distributions.

In addition, the consolidation of much of the integrated modeling practice within a handful of developers/consulting firms arguably tends to impose a conservatism in the field. It is arguable that consultants are often more concerned with maximizing the return on past investments in their models than necessarily incurring new costs and risks to develop substantively new products.

A number of possible reasons for this "stalling" of integrated urban modeling progress exist, including: past failures, the large data and resource requirements to develop an operational model system, lack of resources (money, people, time) within many/most urban planning agencies, "silo-ing" of urban planning and transportation planning in separate agencies or divisions in many municipal governments, reluctance among many local planning agencies to incorporate formal models into their planning processes, distrust of formal models by many qualitatively-oriented planners, lack of mandate within many municipal planning agencies to regulate land development (a common issue, for example, in many US jurisdictions), and, perhaps increasingly, a disdain for evidence-based planning and decision-making 
within many agencies and governments.

Some of these issues are more readily addressable than others by researchers interested in advancing the integrated urban modeling state of practice. But it is also arguable that another barrier to advancing the practice is the relative lack of young researchers taking on the integrated modeling challenge: there is not much evidence to suggest that a younger generation of academics is rushing into the integrated urban modeling field. As evidenced by the WSTLUR conferences, academic research into transport/ land-use interactions is alive and well, but little of this research is being translated into (or is even directly supportive of) large-scale urban modeling. Again, barriers to such involvement are identifiable, including:

- The omnipresent pressure to publish, with more "basic" (theoretical or empirical) papers being preferred by journals (and, frankly, generally easier to produce) than the more "applied" papers emerging from integrated model development.

- The risk and hard work involved in building large-scale operational models; building a working integrated model system involves a large amount of software development, database assembly, nitty-gritty attention to details, model testing, calibration and validation of both model components and the overall, complex model system (as oppose to "just" parameter esti mation of individual models).

- The considerable resources required to build a large-scale model (data, a large research team, time to develop the model system, etc.)

- Difficulty in attracting, on the one hand, granting agency funding for such relatively applied research, and, on the other, government agency funding for such relatively risky R\&D.

- Current operational integrated modeling software systems are very monolithic and often pro prietary. Even if nominally open source, they are sufficiently complex and opaque that they are challenging for individual researchers to use as a research platform or to make contributions to advancing the software.

Despite these many obstacles, as argued in the paper's introduction, the need for useful operational integrated urban models remains. The next section of the paper discusses criteria that "next generation" integrated model systems should address. In particular, it is argued that a key requirement is the development of a common, generic software environment within which individual models and model components can be developed and tested without having to develop a full integrated model system from scratch. Such a software environment would both enable individual researchers with modest resources to contribute to the field and facilitate the "crowd sourcing" of new model systems that could emerge over time. Given this foundation, Section 4 introduces and briefly discusses a range of research topics that need to be addressed if such a next generation model system is to emerge.

\section{Criteria for next generation IUMs}

Miller, et al. (1998) provide a detailed discussion of integrated urban model design criteria, which include:

- Representation of the physical urban system

- Representation of decision-makers

- Representation of decision processes 
- Implementation issues:

- Other "generic" issues:

Data requirements

o Computational requirements

o Technical support requirements

o Aggregation vs. disaggregation representation

o $\quad$ Endogenous vs. exogenous variables

o "Type" of process ("behavioral," "statistical rates," etc.)

o $\quad$ Model specification issues

They also identify criteria for evaluating model systems, which include:

- Credibility (theoretical soundness, policy sensitivity, etc.)

- Feasibility

- Usability (ease of input preparation, run times, portability, etc.)

Nearly 20 years later these guidelines still stand up (if I am permitted to say so!), and so "the interested reader" is referred to the original report rather than trying to reproduce this discussion herein. But it is useful to add a few new observations reflecting current and emerging policy analysis needs and modeling capabilities. The first of these is that the policy context continues to grow more complex. Going back to the dawn of land-use modeling in the 1960s, the initial motivation for developing such models was to generate the population and employment inputs required by regional travel demand models. Beginning in the 1970s the importance of understanding the impact of major transportation investments (particularly transit) on land values was increasingly recognized, as well as the importance of land value impacts on benefit-cost justifications of major (and expensive) transportation infrastructure (again, particularly transit). These core requirements remain today, but additional key policy questions that could benefit from integrated model-based analysis include:

- Designing sustainable urban forms (in both developed and developing countries)

- Equity considerations concerning accessibility (to jobs, services, food, etc.) affordable housing, etc.

- Urban economic development

- Energy, GHG and air pollution impacts

- Health impacts of land-use patterns and travel behavior

Addressing these and similar issues requires a model system that is more than just a "land-use" model. We need to be thinking in terms of an integrated approach to modeling an interconnected set of urban spatial, socioeconomic systems (housing, demographics, firmographics, transportation, etc.) within a systems-of-systems framework.

Continuing methodological advances mean that formerly major barriers to integrated modeling (data, computational requirements, etc.), while still challenging, now represent opportunities for model system development, if they can be exploited. These include:

- Cost-effective High Performance Computing (HPC) options (cloud/cluster computing, parallelization, GPU-based processing, etc.) hold the promise of significantly improving the computational performance of large integrated models.

- Increasingly ubiquitous, detailed GIS-based datasets, notably (but not exclusively) "big" datasets are dramatically improving the databases upon which integrated models can be built.

- Machine-learning and other advanced data analysis and modeling methods are adding very exciting and powerful new tools and approaches to our modeling toolkits.

Specific research questions are discussed in the next section. But a few generic challenges that need to be addressed despite (and in some cases because of) these new capabilities include the following. 
Model parsimony: Integrated urban models typically are very complicated beasts with many hundreds of parameters. Some "complicatedness" in dealing with such a complex system as an urban region is unavoidable. Nevertheless, it is important to ask whether simpler formulations might work as well as or even better than more complicated ones in terms of data requirements, predictive accuracy, computational requirements and understandability (discussed further immediately below). It is argued elsewhere (Miller, 2017), that agent-based microsimulation (ABM) provides a promising framework for dealing in parsimonious ways with complex behavioral processes.

Model transparency: Similarly, the extent to which the workings and the results of an integrated urban model are transparent/understandable/explainable by both the modeler and the users of the model outputs (planners, politicians, the public) is extremely important for the model's usability and credibility. In the author's experience, again, the relative simplicity and intuitive structure of the ABM approach has proven useful in explaining an advanced activity-based travel modeling system to senior municipal decision-makers and in gaining their confidence in the credibility of the model's outputs (Miller, 2015). Deep machine-learning and other AI-based methods appear very promising as new approaches to model development (e.g., among others, Yin, Sheehan, Feygin, Paiement, \& Pozdnoukhov, 2016). But concerns exist concerning the "black box" nature of these models, which may perform well but which may not have readily explainable parameters or model structures.

Model validation: A chronic weakness of many integrated urban modeling efforts has been the inability to validate the model's performance outside of the model's estimation/calibration dataset. This is understandable, up to a point, given the multi-decade nature of a typical model's outputs. Consistent, quality data over such long periods for a given urban region often have not been available to support such a validation. A second validation approach is to transfer a model from its development urban region to a different application region. This generally is an extremely onerous and challenging proposition. The famous ISGLUTI effort in the 1980's to cross-run several land-use models in a range of cities clearly demonstrated the challenges involved in such an exercise (Webster, Bly, Paulley, \& Brotchie, 1988). Even the cross-running of two or more models in a single urban region is challenging (Rodier, 2004; Kockelman \& Krishnamurthy, 2003). A third validation approach is to conduct a wide variety of sensitivity tests of a model to explore the reasonableness and robustness of its performance across a range of out-of-sample applications (Clay \& Johnston, 2005; Wang \& Kockelman, 2016). Regardless of method used, validation is an extremely important component in model development and testing. Many models may "calibrate well" against base conditions, but not perform well in application. It is only by testing a model across a range of applications can its true performance be assessed and confidence in this performance be achieved. The increasingly rich, deep and "big" datasets that are often now available (often as open source) have good promise for permitting much more extensive model testing than has typically been possible in the past.

Model software: Integrated urban models by definition exist within software. To date, this software has always been custom-built for the particular model system being implemented. This inevitably results in the modeling assumptions being "hard-wired" into the software in many ways, with the model (as a mathematical construct) and the software (computer code) essentially having a "one-to-one," "monolithic" relationship. This represents a significant barrier to advancing the state of the art in several respects, including:

- It is often difficult to experiment with new model functional forms, assumptions, etc., since current assumptions are hard-coded into the software and such tests would require (often extensive) software rewrites.

- It is very difficult for researchers to collaborate in terms of bringing new ideas to the table since, again, the software does not readily support implementation of new modules, etc. 
- To the extent that the software is associated with commercial ventures there is often business pressures to use and reuse existing software rather than to invest in new products.

- Even if open source, the software systems are difficult to learn and work with.

- Given the complexity and size of urban regions, developing powerful software is often beyond the resources of individual researchers.

Ideally, modeling software should provide a "virtual lab" within which researchers and model developers can experiment with alternative formulations and test new hypotheses. It should be modular so that new algorithms, modules, etc. can be "plugged into" an overall model system or testing platform. This platform should support such testing in ways that do not impose unwanted assumptions or constraints on the modules being tested. The software environment should also provide a standard "tool kit" for dealing with the many elements of model development, testing and implementation—data input preparation, display and comparison of model outputs, parameter estimation procedures, etc. - so that these elements do not have to be constantly re-invented.

Over the past decade or more there has been some movement within both the land use and transportation modeling communities to try to develop open source software to support model development by multiple users, rather than to implement a specific model by a single development team. Examples include the Open Platform for Urban Simulation (OPUS) developed by the UrbanSim group (Waddell, Sevcikova, Socha, Miller, \& Nagel, 2005), Argonne Lab’s POLARIS project (http://polaris.es.anl. gov/), University of Toronto's eXtensible Travel Modeling Framework (XMTF, http://tmg.utoronto.ca/ doc/1.3/) and the international MATSim consortium (Horni, Nagel, \& Axhausen, 2016). To date, only MATSim has developed a community of users who share results and contribute to growth in the software. But even here, the software requires one to "buy into" the MATSim "paradigm," at least to some degree, and it does not provide the very broad, generalized platform envisioned above.

A number of reasons for the lack of shared software and collaborative model development efforts exist. The choice of programming language is a non-trivial one, with different developers using python, Java, C\#, C++ and R, among probably others. Another, deeper barrier, however, is the "MyModel syndrome." A number of years ago, Frank Southworth, then at Oak Ridges National Lab, surveyed the land-use modeling community. In each interview he asked "and what is the best model?" The universal answer was "why my model, of course." This attitude can readily be extended to the notion of "MySoftware."

It is arguable that the integrated urban modeling state of the art could make much more progress much more quickly — and attract many more researchers into the field — if we could get past this "MyModel/MySoftware" mentality and develop a truly collaborative research environment. This would require:

- Developers to surrender "ownership" of "their" model systems in favor of commonly shared systems within which many researchers can contribute. The MATSim community provides a good example of this approach.

- Creation of open source model development and testing platforms that are "agnostic" with respect to modeling assumptions (as much as possible), and that provide a sharable (and con stantly extendible) set of modeling tools.

- This "virtual lab" should support the head-to-head testing of assumptions, models, etc. so as generate real advances in both behavioral theory and modeling practice. This would require researchers to be willing to put their theories to the test (and to occasionally "fail the test") something that, frankly, rarely occurs in our field at the moment.

- Ideally, sharing of datasets. 


\section{$4 \quad$ Research agenda suggestions for building next generation models}

\section{1 $\quad 4.1 R \& D$ components}

Many major areas for integrated urban modeling R\&D exist. An undoubtedly incomplete list is presented and very briefly discussed below. Summary, overview comments concerning next generation modeling research needs then follow.

Building supply models: While models of housing and (to a somewhat lessor extent) commercial floorspace demand are generally well developed (thanks in large measure to the extensive application of powerful discrete choice modeling methods), housing and commercial floorspace supply models are not nearly so well developed, and, as result, generally not as reliable. Building stock supply modeling is a challenging task given the relatively small number of agents (developers and builders) involved, and, hence, inevitably more idiosyncratic decision-making. Whether for this reason, or others, building stock supply has been a relatively under-researched area.

Choice set definitions: The weak link in building stock demand modeling is typically choice set determination (Miller, 2013b). Despite decades of research (especially with respect to housing markets), the dynamics of spatial search processes are generally not well understood and are typically challenging to observe. Very often quite crude methods (e.g., random choice set generation) are used to generate the choice sets to which much more sophisticated choice models and market clearing procedures are then applied.

Demographics: Most operational models are improving their treatment of household and individual demographics, but, as a general rule, these models still can be significantly improved. Demographics, of course, are critical to the understanding and modeling of housing, labor and travel markets - the key elements of the integrated model system.

Regional Economics: It is increasingly common to frame the modeling of land-use markets and travel demand to a greater or lesser extent within some type of regional economic model (with MEPLAN, Tranus and PECAS being the most notable examples of this approach). This is an attractive approach as a means of providing a unifying conceptual framework for the overall model system, especially with respect to modeling urban goods movements. Much more can be done, however, to develop robust models of regional economic activity and to reduce the data burden associated with this approach. Integrated urban models must, one way or another, do a better job of modeling firmographics (evolution of firms and employment, including employment locations), as well as improved modeling of place of residence-place of work commuting linkages as the explicit outcome of labor market processes.

Auto Ownership: Current model systems generally include at least some representation of household auto ownership decision-making. Again, however, these models generally should be improved. Auto ownership has a major impact on (and interrelationship with) residential and job location choices, as well as, of course, travel behavior. Failure to adequately model the interconnections between auto ownership decisions with residential and employment locations, in particular, could represent an important misspecification of these processes and may represent a significant lost "degree of freedom" in policy analysis. I.e., policies which reduce the number of cars required by households to meet their daily activity needs may be particularly effective in reducing auto dependency and other desired policy goals. This need for improved representation of household auto ownership processes will become even more critical in coming years as new modes of auto ownership/usage (i.e., car-sharing services) become increasingly prevalent and as new auto technologies (autonomous and/or connected vehicles) become available.

Short- \& long-run interactions: All IUMs involve some form of information flow between longerrun land-use (location) choices and short-run trip-making, with the nature of this interaction varying among model systems from a "loose connection" involving "feedback" of transportation accessibility 
terms into location choices (e.g., UrbanSim and Cube Land, among others) to a "strong integration" in which work travel is the explicit outcome of place of residence-place of work location decisions and other travel is the outcome of economic interactions of various types. The way in which day-to-day experiences within the transportation system actually influences longer-run location choices is not, however, well understood. Considerably stronger theory concerning how short-run experiences help shape longrun decisions is needed. This is especially true in terms of developing more truly dynamic integrated models, given the extent to which these models generally are still strongly static in many respects

\subsection{General comments}

The first two of the R\&D areas listed above (improved building stock supply modeling and improved choice set models/procedures) are critical to ensuring the credibility of integrated models. Their fundamental rationale is to predict the spatial distribution over time of people and jobs. If, however, they cannot adequately predict where new building stock (and how much and of what type) will be built, and/ or predict where households and firms of different types will locate/relocate given the built urban form, then the models simply will not be credible.

The second two R\&D areas illustrate that current and emerging model systems are (and need to be) much more than "land-use" models. To varying degrees, they are developing into more comprehensive models of "spatial socio-economic processes" and are becoming as much general models of regional economics and demographics as they are models of "just" population and employment distributions. This trend further generalizes to thinking of these model systems as the foundation upon which other spatial systems can be overlaid such as energy, water, waste and communication systems and/or "social" systems such as education, health care, etc. to build a "systems of systems" representation of urban regions.

Further, we are rapidly entering an era of "complete mobility systems" and potential autonomous vehicle deployments that will require us to rethink our concepts of both accessibility and mobility and that may well alter in unforeseen ways the transportation/land-use interaction. If our integrated urban models are to remain useful as policy analysis tools in the light of these potential disruptive technologies they must be sufficiently fundamental in their behavioral foundations and sufficiently flexible/extensible in their methodological and computational implementations to accommodate these changes. Whether the current generation of operational models are up to this challenge remains to be seen. It is hoped, however, that they will continue to evolve, both in their theoretical foundations and their practical implementations in ways that will permit them to adapt appropriately. To this end, as noted above, two very different modeling approaches both offer interesting ways forward. Agent-based formulations arguably provide an attractive implementation framework given their potential flexibility for implementing behaviorally sound models of adaptive choice. At the same time, machine-learning methods are well suited for exploiting big datasets in new and interesting ways. Whether these two approaches can prove to be mutually reinforcing in some way is an interesting research question in its own right.

Finally, we are gradually achieving new insights into cities as complex systems that seem to obey macro-level scaling laws, fractal behavior, etc. (Batty, 2005, 2008, 2013; Bettencourt, Lobo, Helbing, Kuhnert, \& West, 2007; Bettencourt \& West, 2010; Bettencourt, 2013). The implications (if any) for integrated urban modeling, or, conversely, how integrated models might contribute to this emerging "science of cities," is far from clear, but, again, a question worthy of study.

\section{$5 \quad$ Final comments}

Greater future usage of integrated models depends on being able to demonstrate their practicality and usefulness within the planning process. The "first generation" of land -use modeling in the 1960's (Gold- 
ner, 1971) came to a virtual halt in the 1970's due to widespread dissatisfaction with the failure of these models to meet their decision-support goals (Lee, 1973) — a setback from which it has taken the field literally decades to recover. As argued in this paper, improved operational performance of these models depends in the first instance on significantly improving their theoretical and empirical foundations.

This, arguably, may be most likely to happen if a new generation of researchers can be motivated to take up this task. In turn, if this is to happen, then both barriers to engagement need to be removed and incentives for engagement need to be provided. Creating a powerful, open source, commonly accessible software environment-a virtual lab-within which researchers could use common tools and (whenever possible) common datasets within an "agnostic" modeling environment would remove many resource barriers. It would also provide a virtual environment within which a community of like-minded researchers could network, interact and collectively advance the state of knowledge and art in the field. It would help break down the "MyModel/MySoftware" syndrome and allow researchers to "constructively compete" in terms of positing and testing alternative theories, models and model systems. The next, improved generation of integrated urban models would then hopefully emerge out of the nexus of the best ideas and methods that were collectively developed within the virtual lab and its associated research community.

\section{Acknowledgments}

The research underlying this paper has been supported in part by Canada Natural Science and Engineering Research Council Discovery and Accelerator research grants, as well as by an Ontario Research Fund, Research Excellence Round 7 grant. 


\section{References}

Batty, M. (2005). Cities and complexity: Understanding cities with cellular automata, agent-based models and fractals. Cambridge MA: MIT Press.

Batty, M. (2008). The size, scale and shape of cities. Science, 319, 769-771.

Batty, M. (2013). The new science of cities. Cambridge MA: MIT Press

Bettencourt, L. M. A., Lobo, J., Helbing, D., Kuhnert, C., \& West, G. B. (2007). Growth, innovation, scaling, and the pace of life in cities. Proceedings of the National Academy of Science, 104(17), 7301-7306.

Bettencourt, L. M. A., \& West, G. B. (2010). A unified theory of urban living. Nature, 467, 912-913.

Bettencourt, L. M. A. (2013). The origins of scaling in cities. Science, 340, 1438-1441.

Clay, M. J., \& Johnston, R. A. (2005). Univariate uncertainty analysis of an integrated land use and transportation model: MEPLAN. Transportation Planning and Technology, 28(3), 149-165.

de la Barra, T. (1989). Integrated land use and transport modeling: Decision Chains and hierarchies. Cambridge, United Kingdom: Cambridge University Press.

Demographia. (2017). Demographia world urban areas, 13th annual edition. Retrieved from http:// demographia.com/db-worldua.pdf

Goldner, W. (1971). The Lowry model heritage. Journal of the American Institute of Planners, 37(2), $100-110$.

HBA Specto. (2013). Best practice review of integrated land use - transportation models, literature review, project technical report 1. Calgary, Alberta: Ontario Ministry of Transportation.

Horni, A., Nagel, K., \& Axhausen, K. W. (2016). The multi-agent transport simulation MATSim. Retrieved from http://matsim.org/the-book

Kockelman, K., \& Krishnamurthy, S. (2003). Propagation of uncertainty in transportation-land use models: Investigation of DRAM-EMPAL and UTPP predictions in Austin, TX. Transportation Research Record, 1831, 219-229.

Lee, D. B. (1973). Requiem for large scale models. Journal of the American Institute of Planners, 39, $163-178$.

Lee, D. B. (1994). Retrospective on large scale urban models. Journal of the American Planning Association, 60, 35-40.

Martinez, F. J. (1992). The bid-choice land-use model: An integrated econometric framework. Environment and Planning A, 24, 871-875

Miller, E. J. (2009). Integrated urban models: Theoretical prospects, invited resource paper. In R. Kitamura, T. Yoshii, \& T. Yamamoto (eds.), The expanding sphere of travel behavior research: Selected papers from the 11th international conference on travel behavior research (pp. 351-384). Bingley, UK: Emerald.

Miller, E. J. (2013a). Towards a third urban revolution: Transportation and cities in the 21 st century. Margolese National Prize for Urban Design acceptance lecture, Vancouver, March 18.

Miller, E. J. (2013b). Spatial choice modeling issues. Presented at TRISTAN VII, San Pedro de Atacama, Chile, June 9-14.

Miller, E. J. (2015). First, we take Toronto...lessons from the TASHA implementation and next steps. Invited presentation, Behavioral detail and computational demands in agent-based models workshop, Future Cities Laboratory, Singapore, March 24-26.

Miller, E. J. (2017). The case for microsimulation frameworks for integrated urban models. Submitted to Journal of Transport and Land Use.

Miller, E. J., Kriger, D. S., \& Hunt, J. D. (1998). Integrated urban models for simulation of transit and 
land-use policies, final report of the Transit Cooperative Research Project H-12. Toronto: University of Toronto Joint Program in Transportation.

Moeckel, R. (2017). Constraints in household relocation: Modeling land-use/transport interactions that respect time and monetary budgets. Journal of Transport and Land Use, 10(2), 1-18.

Moeckel, R., Schwarze, B., Spiekermann, K., \& Wegener, M. (2007). Simulating interactions between land use, transport and environment. Proceedings of the 11th World Conference on Transport Research, Berkeley, CA: University of California at Berkeley.

Pas, E. I. (1990). Is travel demand analysis and modeling in the doldrums? In P.M. Jones (ed.) Developments in dynamic and activity-based approaches to travel analysis (pp. 3-27). Farnham, United Kingdom: Aldershot.

Rodier, C. J. (2004). Verifying the accuracy of regional models used in transportation and air quality planning. Transportation Research Record, 1898, 45-51.

Rosenfield, A., Chingcuanco, F., \& Miller, E. J. (2013). Agent-based housing microsimulation for integrated land use, transportation, environment model system. Procedia Computer Science, 19, 841846.

Spiekermann, K., \& Wegener, M. (2007). The PROPOLIS model for assessing urban sustainability. In M. Deakin, G. Mitchell, P. Nijkamp, \& R. Vreeker (eds.) Sustainable urban development, volume 2, the environmental assessment methods (pp. 306-326). Abingdon, United Kingdom: Routledge.

Strauch, D., Moeckel, R., Wegener, M., Gräfe, J., Mühlhans, H., Rindsfüser, G., \& Beckmann, K.-J. (2005). Linking transport and land use planning: The microscopic dynamic simulation model ILUMASS. In P. M. Atkinson, G. M. Foody, S. E. Darby, \& F. Wu (eds.), Geodynamics (pp 295-311). Boca Raton, Florida: CRC Press.

Timmermans, H. (2003). The saga of integrated land use-transport modeling: How many more dreams before we wake up? Conference keynote paper, 10th International Conference on Travel Behavior Research, Lucerne, Switzerland.

Waddell, P., Sevcikova, H., Socha, D., Miller, E. J., \& Nagel, K. (2005). Opus: An international collaboration to develop an open platform for urban simulation. Presented at the CUPUM Conference, London, June 29-July 1.

Wang, G., \& Kockelman, K. (2016). Methods for uncertainty propagation in transportation and land use models. Forthcoming in Journal of the Transportation Research Forum.

Webster, F. V., Bly, P. H., Paulley, N. J., \& Brotchie, J. F. (1988). Urban land-use and transport interaction: Policies and models, report of the International Study Group on Land-Use/Transport Interaction (ISGLUTI). Farnham, England: Aldershot.

Wegener, M. (1995). Current and future land-use models. In G.A. Shunk, P. L. Bass, C. A. Weatherby, and L. J. Engelke (eds.), Travel Model Improvement Program Land-Use Modeling Conference Proceedings, 13-40. Washington, DC: Travel Model Improvement Program.

Wegener, M. (2011). The IRPUD model, working paper 11/01. Dortmund, Germany: Spiekermann and Wegener Urban and Regional Research.

Weidner, T., Abraham, J. E., Donnelly, R., Hunt, J. D., \& Freedman, J. (2007-January). A summary of the Oregon TLUMIP model microsimulation modules. Presented at the 2007 Annual Meeting of the Transportation Research Board, Washington, DC.

Yin, M., Sheehan, M., Feygin, S., Paiement, J.-F., \& Pozdnoukhov, A. (2017). A generative model of urban activities from cellular data. IEEE Early Access Articles, 1-15 